\title{
DETERMINACIÓN DE LA EDAD SUSCEPTIBLE DEL FRUTO DE LA PAPAYA (Carica papaya L.) A LA ANTRACNOSIS (Colletotrichum gloeosporioides. (PENZ.)) ${ }^{1}$
}

\author{
Alfredo Durán², Dennis Mora², Erick Chavarría ${ }^{3}$
}

\begin{abstract}
RESUMEN
Determinación de la edad susceptible del fruto de la papaya (Carica papaya L.) a la antracnosis (Colletotrichum gloeosporioides. (Penz.)). Con la finalidad de determinar las etapas de susceptibilidad de la papaya criolla a la antracnosis del fruto producida por Colletotrichum sp. , así como para comprobar la presencia de genotipos resistentes a esta enfermedad, se realizó una investigación en la zona de Sarapiquí , provincia de Heredia, durante el período comprendido entre mayo y setiembre de 1993. Los resultados observados indican que los frutos de todas las edades evaluadas, fueron susceptibles a la antracnosis y que el combinado de genotipos de papaya criolla puede presentar dos situaciones: manifestar resistencia permanente a la enfermedad o no manifestarla del todo. No se encontraron evidencias de que el fruto pueda manifestar una resistencia temporal, durante alguna etapa de su período de crecimiento, que sea enmascarada por posteriores períodos susceptibles. Tampoco se encontraron evidencias que permitan sugerir etapas en los frutos de mayor o menor susceptibilidad a la enfermedad. En algunos genotipos de papaya criolla se encontró resistencia durante todo el período evaluado.
\end{abstract}

\begin{abstract}
Determination of susceptible age of papaya (Carica papaya $\mathrm{L}$ ) to anthracnose (Colletotrichum gloeosporioides. (Penz.). A research was carried out in Sarapiquí, Heredia, Costa Rica, between May and September, 1993. The objective was to determine the phases of susceptibility of native papaya to anthracnose produced by Colletotrichum sp., as well as to prove the presence of anthracnose-resistant genotypes. Results indicated that at all ages, fruits examined were susceptible to anthracnose, and that a combination of native papaya genotypes may either present a permanent resistance to the disease, or never show any signs at all. There was no evidence to prove that fruits might show a temporary resistance at certain development phases, which might be camouflaged by subsequent susceptible periods. Nor was there evidence to suggest phases of greater or lesser susceptibility to the disease. Some native papaya genotypes showed resistance through the period examined.
\end{abstract}

\section{INTRODUCCIÓN}

La antracnosis del fruto producida por Colletotrichum gloeosporiodes Penz. sp. es la enfermedad poscosecha más importante de la papaya (Carica papaya L.) en Costa Rica (Arauz y Mora 1983).

El manejo de la enfermedad es cada día más difícil, debido a que se deben cumplir estrictas normas de calidad para la fruta de exportación y a la vez existen regulaciones técnicas en cuanto a residuos de agroquí- micos en el fruto, uso racional de fungicidas sistémicos para evitar la generación de resistencia y la protección del ambiente. Entre otras, para el manejo de la enfermedad existen dos estrategias con amplias posibilidades de desarrollarse, las cuales representan opciones sostenibles para el combate del hongo: la resistencia permanente del fruto al ataque del hongo y el manejo del combate, dirigiéndolo únicamente a la etapa de desarrollo del fruto susceptible al ataque de la enfermedad, en caso de que esta condición se presente para alguna variedad.

\footnotetext{
1 Proyecto mejoramiento de la calidad poscosecha de la papaya mediante métodos complementarios de campo. Vicerrectoría de Investigación, Estación Experimental Fabio Baudrit. Universidad de Costa Rica.

2 Laboratorio de Fitopatología CIPROC/Universidad de Costa Rica.

3 Liga Agrícola Industrial de la Caña de Azúcar, LAICA.
} 
Al respecto Durán y Mora (1987) en observaciones realizadas sobre una combinación de genotipos de papaya criolla en Costa Rica, encontraron en diversas ocasiones frutos resistentes a la antracnosis, al no presentar daños por el hongo, aún cuando frutos de la misma edad y procedentes de la misma plantación, mostraron diferentes intensidades del daño producido por Colletotrichum sp.

En Costa Rica no se han realizado estudios sobre combinados de genotipos de papaya tipo criollo, que demuestren claramente la presencia de materiales genéticos con resistencia permanente o temporal, al ataque de la antracnosis del fruto.

Con referencia a la resistencia temporal de los frutos de papaya durante su proceso de desarrollo, Stanghellini y Aragaki (1966), trabajando con papaya de la línea "Solo" informaron que la antracnosis sólo puede afectar frutos mitóticamente inactivos, razón por la cual los síntomas se presentan sólo en las últimas etapas del desarrollo del fruto.

Posteriormente Dickman y Alvarez, (1983) bajo condiciones de campo, comprobaron la infección de frutos del cultivar Kapoho-Solo por Colletotrichum sp. durante todo su desarrollo, así como la presencia de infecciones latentes del hongo.

Informes similares han sido presentados por diversos autores, trabajando siempre con líneas de papayas desarrolladas a partir de materiales de origen hawaiano.

La presente investigación se realizó con el fin de determinar con precisión las etapas de susceptibilidad de un combinado de genotipos de papaya de tipo criollo a la antracnosis del fruto producida por Colletotrichum sp. y además comprobar la presencia de genotipos resistentes a la antracnosis dentro de las poblaciones de material nativo.

\section{MATERIALES Y MÉTODOS}

La investigación se realizó en los meses de mayo a setiembre de 1993 en la localidad de San Rafael de la Virgen de Sarapiquí en la provincia de Heredia, Costa Rica, ubicada entre los paralelos $10^{\circ}$ y $10^{\circ} 30^{\prime}$ y los meridianos $80^{\circ}$ y $80^{\circ} 30^{\prime}$; por ser una zona cuyo clima permite la producción de papaya y la vez favorece el desarrollo de la enfermedad. El área donde se establecieron los experimentos de campo pertenece a la zona de vida Bosque muy Húmedo pre-montano transición a basal.
Los experimentos se realizaron en una plantación comercial de papaya criolla de un año de edad, con una gran diversidad de características tanto del árbol como de la fruta. El cultivo recibió el manejo usual que acostumbran los agricultores de la zona, caracterizado por fertilización bimensual con nitrógeno, fósforo y potasio, control manual de malezas y aplicaciones periódicas de fungicidas a base de benomil y mancozeb.

Dentro de la plantación se encontraron frutos, tanto provenientes de flores hembras como de flores hermafroditas, con sus diferentes variantes de forma como son los llamados cacho corto y cacho largo, así como bombas o frutas redondas. Igualmente se encontraron frutas de pulpa amarilla o roja, con sus colores intermedios, de cavidad redonda o en forma de estrella y con espesor de pulpa de grueso a delgado.

\section{Prueba de inoculación natural controlada.}

Para realizar el experimento se marcaron al azar en la plantación 120 frutos con un tamaño menor a los $10 \mathrm{~cm}$ de longitud, lo que equivale a una edad de 15 días después de la polinización, dicho estado de desarrollo coincide con el desprendimiento de los pétalos. Los frutos marcados fueron ordenados aleatoriamente en 12 grupos de 10 frutos. Cada grupo constituyó un tratamiento.

Con el fin de evaluar la susceptibilidad del fruto al hongo Colletotrichum sp en distintos períodos, se les colocó una bolsa perforada de polietileno transparente a 11 grupos de frutas en su extremo inferior, de tal manera que permitiera su crecimiento aislado del inóculo natural de la plantación, en períodos conocidos según el tratamiento respectivo. La fruta estuvo expuesta al inóculo natural de campo cuando los diversos tratamientos estuvieron sin bolsa.

A seis grupos de frutas se les colocó la bolsa de polietileno desde el inicio y se les aplicó un tratamiento que consistía en retirarle la bolsa a los 28, 42, 56, 70 y 84 días después de la polinización. El sexto grupo permaneció embolsado hasta la cosecha.

A otros cinco grupos marcados se les colocó la bolsa de polietileno transparente, usando el mismo procedimiento a partir de los $28,42,56,70$ y 84 días después de la polinización y se les dejó embolsados hasta la cosecha. El último grupo de frutos o tratamiento testigo, permaneció sin bolsa hasta la cosecha y no se le aplicó ningún tratamiento. 
En el Cuadro 1 se resumen las características de los diferentes tratamientos utilizados durante el período del experimento. Para la protección del fruto se suspendieron las aplicaciones de fungicidas.

\section{Prueba control 1. Inoculaciones artificiales.}

Con el fin de prever la detección del efecto que podría tener un escape de las frutas de un tratamiento, como consecuencia de una condición ambiental adversa, se realizó en forma paralela al experimento de inoculaciones naturales controladas, un experimento de inoculaciones artificiales a grupos de 10 frutos de 15 , 30, 45, 60 y 75 días de edad. La inoculación se hizo con una suspensión de aproximadamente 104 esporas provenientes de un cultivo de Colletotrichum sp. aislado de frutos enfermos de la misma finca. Para la inoculación se aplicó mediante aspersión y sin realizar heridas, un mililitro de la suspensión de esporas sobre la superficie del fruto, posteriormente se colocó una bolsa de polietileno transparente por 48 horas para formar una cámara húmeda. Además, se dejó un tratamiento testigo al cual se le aplicó sólo agua estéril. Las inoculaciones se hicieron sólo en la mitad inferior del fruto, a fin de diferenciar sus síntomas de los producidos por el inóculo de campo. Para ello durante las inoculaciones

Cuadro 1. Características de los tratamientos probados en la Prueba de Inoculación Natural Controlada de frutos de papaya (Carica papaya L.) durante toda sus etapas de desarrollo. Sarapiquí, Heredia, Costa Rica, 1993.

\begin{tabular}{|c|c|c|c|c|}
\hline \multicolumn{2}{|c|}{ Tratamiento } & \multirow{2}{*}{$\begin{array}{c}\begin{array}{c}\text { Días } \\
\text { embolsado }\end{array} \\
28 \text { a cosecha }\end{array}$} & \multirow{3}{*}{\begin{tabular}{l}
\multicolumn{1}{c}{$\begin{array}{c}\text { Días sin } \\
\text { bolsa }^{1}\end{array}$} \\
14 a 28 \\
28 a cosecha
\end{tabular}} & \multirow{2}{*}{$\frac{\begin{array}{c}\text { Etapa } \\
\text { evaluada } 1\end{array}}{14 \text { a } 28}$} \\
\hline 2 & A & & & \\
\hline & B & 14 a 28 & & 28 a cosecha \\
\hline \multirow[t]{2}{*}{4} & A & 42 a cosecha & 14 a 42 & 14 a 42 \\
\hline & B & 14 a 42 & 42 a cosecha & 42 a cosecha \\
\hline \multirow[t]{2}{*}{6} & A & 56 a cosecha & 14 a 56 & 14 a 56 \\
\hline & B & 14 a 56 & 56 a cosecha & 56 a cosecha \\
\hline \multirow[t]{2}{*}{8} & A & 70 a cosecha & 14 a 70 & 14 a 70 \\
\hline & B & 14 a 70 & 70 a cosecha & 70 a cosecha \\
\hline \multirow[t]{2}{*}{10} & A & 84 a cosecha & 14 a 84 & 14 a 84 \\
\hline & B & 14 a 84 & 84 a cosecha & 84 a cosecha \\
\hline \multirow{2}{*}{\multicolumn{2}{|c|}{$\begin{array}{l}\text { Embol Total } \\
\text { Testigo }\end{array}$}} & 14 a cosecha & 0 a 14 & 0 a 14 \\
\hline & & 0 & 0 a cosecha & 0 a cosecha \\
\hline
\end{tabular}

1 Días después de la polinización. se aisló la mitad superior del fruto mediante una bolsa plástica adherida con una banda de hule.

\section{Prueba control 2.}

Con el objetivo de llevar un control de la presencia del inóculo natural de campo de Colletotrichum sp., se hicieron observaciones quincenalmente sobre los pecíolos de las hojas senescentes aún adheridos al árbol y sobre los pétalos necrosados de flores recién polinizadas todavía adheridas a la planta. El material se empacó en bolsas plásticas y se llevó al laboratorio en donde se colocó en cámara húmeda. Dos días después se hizo un raspado de signos y se identificaron las esporas presentes.

Para la evaluación de los resultados, se cosecharon los frutos cuando presentaron el estado de madurez, correspondiente al punto de corte de la papaya de tipo criollo. Dicho estado se caracteriza por la presencia de una banda amarilla, la cual muestra el inicio de la madurez en una cara de la mitad inferior del fruto.

Una vez cosechados los frutos se pusieron en incubación hasta la madurez total y se determinó la presencia de la antracnosis mediante la observación de síntomas, el raspado de esporas y la observación al microscopio.

En el caso de las inoculaciones artificiales (Prueba control 1) además, se realizaron aislamientos. En ambas pruebas se evaluó la presencia de la enfermedad mediante el promedio de días a síntomas (PDS), la mayor edad de un fruto para manifestar síntomas (MAES) y la menor edad del fruto para manifestar síntomas (MEES).

\section{RESULTADOS Y DISCUSIÓN}

Durante todo el período la temperatura máxima y mínima estuvo dentro del rango óptimo para el desarrollo de la infección de Colletotrichum sp. en frutos de papaya, determinados por Gupta y Pathak (1990). Los promedios fueron de 33 y $19,9^{\circ} \mathrm{C}$ para la máxima y la mínima respectivamente y de $22^{\circ} \mathrm{C}$ para el promedio general. De igual forma los niveles mínimos y máximos de precipitación estuvieron entre los 306,1 y 420,7 mm por mes, lo cual garantizó la diseminación natural del hongo.

En el Cuadro 2 se presentan los resultados de las observaciones de patógenos presentes en las flores y pecíolos senescentes de la plantación, cuando se realizaron los experimentos de campo. 
Cuadro 2. Hongos observados en flores senescentes y pecíolos de papaya criolla (Carica papaya L.) durante el período de desarrollo de los frutos usados en la Prueba de Inoculación Natural Controlada y en la Prueba Control 1. Sarapiquí, Heredia, Costa Rica 1993.

\begin{tabular}{|c|c|c|c|c|c|c|}
\hline \multirow{2}{*}{$\begin{array}{l}\text { Edad del } \\
\text { fruto }^{1}\end{array}$} & \multicolumn{3}{|c|}{ Hongos observados en flores } & \multicolumn{3}{|c|}{ Hongos observados en pecíolos } \\
\hline & Fusarium sp. & Colletotrichum sp. & Fusarium sp. & Colletotrichum & sp. Corynespora sp. & Alternaria sp. \\
\hline 14 & $\mathrm{X}^{2}$ & $\mathrm{X}$ & & $\mathrm{X}$ & & \\
\hline 28 & $\mathrm{X}$ & $\mathrm{X}$ & & $\mathrm{X}$ & & \\
\hline 42 & $\mathrm{X}$ & $\mathrm{X}$ & & $\mathrm{X}$ & & \\
\hline 56 & $\mathrm{X}$ & & & $\mathrm{X}$ & & \\
\hline 70 & $\mathrm{X}$ & & & $\mathrm{X}$ & & \\
\hline 84 & $\mathrm{X}$ & $\mathrm{X}$ & $\mathrm{X}$ & $\mathrm{X}$ & $\mathrm{X}$ & $\mathrm{X}$ \\
\hline 98 & $\mathrm{X}$ & & $\mathrm{X}$ & $\mathrm{X}$ & & \\
\hline
\end{tabular}

1 Días después de la polinización.

2 La letra X significa que se comprobó la presencia del hongo.

Durante todas las semanas en que se desarrollaron las pruebas, de inoculación natural controlada y las de inoculaciones artificiales (Prueba Control 1), se encontró inóculo de los hongos Fusarium sp. y Colletotrichum sp. tanto en flores como en pecíolos.

En el Cuadro 2 se comprueba una situación normal en las plantaciones de papaya al no recibir un adecuado manejo del inóculo, residuos de cosecha y tejidos senescentes. Durán y Mora (1988) ya habían informado de la presencia de Colletotrichum sp. y Fusarium sp. en pecíolos de papaya secos o senescentes, en Costa Rica.

Los resultados de estas observaciones garantizan la presencia de inóculo de Colletotrichum sp. durante todo el período en que se evaluó la susceptibilidad del fruto a su ataque.

En el Cuadro 3 se presentan los resultados de las pruebas de inoculaciones artificiales (Prueba Control 1), que se desarrollaron en forma paralela a la prueba de inoculación natural controlada.

Los resultados indican que bajo condiciones de alta presión de inóculo (aproximadamente 104 esporas por $\mathrm{ml}$ ), la gran mayoría de los frutos fueron susceptibles al hongo Colletotrichum sp., sin importar la edad cuando se inocularon. Como es usual en las plantaciones de papaya criolla en Costa Rica, según lo informaron Durán y Mora en 1987, se encontró una baja proporción de frutos resistentes a las inoculaciones realizadas. De igual forma, en el tratamiento testigo inoculado con sólo agua estéril, también aparecieron frutos resistentes al ataque del hongo, el cual pudo haber llegado únicamente por la vía del inóculo dispersado en forma natural. Las observaciones de PDS así como MAES y MEES respectivamente, demuestran una gran variabilidad, como es de esperar cuando se trabaja con papaya criolla.

Los resultados obtenidos en esta prueba control también cumplen con el objetivo de proveer información necesaria para eliminar en la prueba de inoculación natural controlada, la posibilidad de que una infección temprana enmascare una etapa posterior de resistencia durante el desarrollo del fruto, tal y como sugiere Stangellini y Aragaki (1966).

Los resultados de la prueba de inoculación natural controlada de frutos (Cuadro 4) demuestran que el combinado de genotipos de papaya criolla puede presentar dos situaciones: los frutos manifiestan resistencia permanente a la enfermedad, o no la manifiestan del todo. Esto por cuanto, no se encontraron evidencias de que el fruto pueda manifestar una resistencia temporal, durante alguna etapa de su período de crecimiento, la cual sea enmascarada por períodos susceptibles.

Los datos del Cuadro 4 indican también, al igual que la Prueba Control 1, que en el combinado de genotipos de papaya criolla, aparece un porcentaje bajo de frutos resistentes a la antracnosis producida por el hongo Colletotrichum sp. Esto coincide con lo encontrado por Durán y Mora en 1987. Al analizar ambas pruebas, se concluye que existieron pocas posibilidades de que estos frutos, que se comportaron como resistentes a la antracnosis sean "escapes" a la enfermedad, puesto que se desarrollaron en presencia comprobada de inóculo y porque las inoculaciones artificiales de la Prueba Control 1, demuestran la susceptibilidad de la mayoría de los frutos en todas sus etapas de desarrollo.

El análisis de la variabilidad de los datos de PDS, así como MAES y MEES respectivamente, para cada 
Cuadro 3. Resultados de la Prueba Control 1 de inoculaciones artificiales de Colletotrichum sp. realizadas durante todo el proceso del desarrollo de frutos de papaya criolla (Carica papaya L.). Sarapiquí, Heredia, Costa Rica, 1993.

\begin{tabular}{lcccccc}
\hline Edad de inocul $^{1}$ & $\begin{array}{c}\text { Edad } \\
\text { evaluada }\end{array}$ & $\begin{array}{c}\text { \% frutos con } \\
\text { antracnosis }\end{array}$ & $\begin{array}{c}\text { \% frutos } \\
\text { sanos }\end{array}$ & P.D.S. ${ }^{2}$ & M.A.E.S. ${ }^{3}$ & M.E.E.S.4 $^{\text {(n) }}$ \\
\hline 14 & 14 & 87 & 13 & 76,1 & 87,5 & 77 \\
28 & 28 & 83 & 17 & 84,0 & 105,0 & 77 \\
42 & 42 & 100 & 0 & 86,9 & 98,0 & 77 \\
56 & 56 & 100 & 0 & 68,4 & 119,0 & 70 \\
70 & 70 & 100 & 0 & 80,2 & 104,3 & 63 \\
Testigo & - & 66 & 33 & 85,8 & 112,0 & 77 \\
\hline
\end{tabular}

1 Edad a partir de la polinización de la flor.

2 P.D.S. Promedio de días a síntomas.

3 M.A.E.S. : Mayor edad del fruto para manifestar síntomas.

4 M.E.E.S. : Menor edad del fruto para manifestar síntomas.

Cuadro 4. Resultados de la Prueba de Inoculación Natural Controlada con el hongo Colletotrichum sp en distintas etapas del desarrollo de frutos de papaya criolla. (Carica papaya L.) Sarapiquí, Heredia, Costa Rica, 1993.

\begin{tabular}{|c|c|c|c|c|c|c|c|}
\hline \multicolumn{2}{|c|}{ Tratamiento Et } & \multirow{2}{*}{$\begin{array}{c}\text { Et. Evaluada } \\
\text { enfer }^{2}\end{array}$} & \multirow{2}{*}{$\begin{array}{c}\begin{array}{c}\% \text { frutos } \\
\text { sanos }\end{array} \\
100\end{array}$} & \multirow{2}{*}{$\begin{array}{c}\% \text { frutos } \\
0\end{array}$} & \multirow{2}{*}{$\begin{array}{r}\text { P.D.S. } \\
123.2\end{array}$} & \multirow{2}{*}{$\frac{\text { M.A.E.S. }}{}{ }^{3}$} & \multirow{2}{*}{$\begin{array}{c}\text { M.E.E.S. }{ }^{\mathbf{4}} \\
70,0\end{array}$} \\
\hline Emb total & 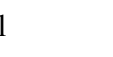 & & & & & & \\
\hline 2 & A & $14-28$ & 100 & 0 & 109,2 & 147,0 & 63,0 \\
\hline & B & $28-\cos 5$ & 100 & 0 & 131,0 & 133,0 & 126,0 \\
\hline \multirow[t]{2}{*}{4} & A & $14-42$ & 100 & 0 & 112,0 & 147,0 & 91,0 \\
\hline & B & $42-\cos$ & 80 & 20 & 106,1 & 133,0 & 77,0 \\
\hline \multirow[t]{2}{*}{6} & A & $14-56$ & 100 & 0 & 110,8 & 147,0 & 98,0 \\
\hline & B & $56-\cos$ & 100 & 0 & 104,1 & 175,0 & 77,0 \\
\hline \multirow[t]{2}{*}{8} & A & $14-70$ & 70 & 30 & 87,5 & 98,0 & 70,0 \\
\hline & $\mathrm{B}$ & $70-\cos$ & 90 & 10 & 137,3 & 175,0 & 84,0 \\
\hline \multirow[t]{2}{*}{10} & A & $14-84$ & 100 & 0 & 133,0 & 151,0 & 91,0 \\
\hline & B & $84-\cos$ & 100 & 0 & 120,7 & 147,0 & 91,0 \\
\hline Testigo & $0-\cos$ & os 100 & 0 & 111,2 & 168,0 & 77,0 & \\
\hline
\end{tabular}

1 Etapa evaluada : Días después de la polinización.

$2 \%$ de frutos enfermos por antracnosis.

3 M.A.E.S. : Mayor edad del fruto para manifestar síntomas.

4 M.E.E.S. : Menor edad del fruto para manifestar síntomas.

5 cos : a la cosecha.

tratamiento, demuestra que no es posible identificar etapas de mayor o menor susceptibilidad en el desarrollo de los frutos. Por otra parte se observa la influencia que tiene una población de hospederos altamente heterogénea sobre el comportamiento de la enfermedad. Esto fuese ñalado por Durán y Mora (1987 ) para materiales criollos de papaya en Costa Rica.
Tanto en la Prueba Control 1, como en el experimento de inoculaciones naturales controladas, la edad mínima cuando el fruto de papaya criolla manifestó síntomas correspondió a los 63 días después de la polinización; bajo las condiciones en que se trabajó.

Estos resultados coinciden con lo manifestado por Dickman y Alvarez (1983), en cuanto a la presencia de 
infecciones latentes de Colletotrichum sp en papaya, hasta que el fruto provea las condiciones necesarias para el desarrollo de las lesiones.

\section{LITERATURA CITADA}

ARAUZ, L.F.; MORA, D. 1983. Evaluación preliminar de los problemas postcosecha en seis frutas tropicales de Costa Rica. Agronomía Costarricense 7 (1/2 ) : 43-53.

DICKMAN, M.B.; ALVAREZ, A.M. 1983. Latent infection of papaya caused by Colletotrichum gloeosporiodes. Plant Disease 67 (7): 748 -750.
DURAN, J.A.; MORA D. 1987. Variabilidad de la sintomatología de la antracnosis (Colletotrichum sp.) en papaya durante el período poscosecha en Costa Rica. Agronomía Costarricense 11 (2) : 155-161.

DURAN J.A.; MORA D. 1988. Diagnóstico de las enfermedades postcosecha de la papaya en Costa Rica: II. Cuantificación y epidemiología de las enfermedades del fruto. Agronomía Costarricense 12 (1) : 7-18.

GUPTA A.K. ; PATHAK, V.N. 1990. Epidemiology and management of papaya fruit rots. Summa Phytophatologica 16: 93- 105 .

STANGHELLINI, M.E.; ARAGAKI, M. 1966. Relation of periderm formation and callose deposition to anthracnose resistance in papaya fruit. Phytopathology 56: 444-449. 